\title{
Mode determination in variational mode decomposition and its application in fault diagnosis of rolling element bearings
}

\author{
P. S. Ambika' ${ }^{10}$ - P. K. Rajendrakumar ${ }^{1} \cdot$ Rijil Ramchand $^{1}$
}

(c) Springer Nature Switzerland AG 2019

\begin{abstract}
The non-stationary nature of bearing vibrations makes it difficult to extract features from the real-time signature of faulty rolling element bearings (REBs). The present work suggests to improve the diagnostic accuracy of fault detection in REBs by enhancing the mode selection property of variational mode decomposition, manipulating its initialization and input parameters (bandwidth selection parameter, $\alpha$ and the number of modes, $k$ ) and then extracting energy entropy features and using cross-validation in support vector machine (SVM) classifier. The alpha values and the number of modes are varied from $(100,1000000)$ and $(4,10)$, respectively. Mean absolute error (MAE) is used as the indicator which calculates error values obtained between the respective sum of modes and the original signal. The particular $\alpha-k$ combination with the least MAE value is chosen. The above method is tested using REB signals obtained from the bearing prognostics test rig. The results obtained from the proposed approach shows $100 \%$ diagnostic accuracy in detecting faulty REB vibration signature using five-fold cross-validation in SVM classifier.
\end{abstract}

Keywords Classification · Cross-validation · Energy entropy · Rolling element bearing · Variational mode decomposition

\section{Introduction}

Rolling element bearings (REBs) reduce the friction between rotating components and are vital for the efficient functioning of a machine. Extreme vibration, a large amount of heat, noise and destruction of metal are some of the warning signs that predict a failure [1]. There are various techniques to detect faults in REBs. Vibration monitoring, acoustic emission monitoring, oil analysis, wear debris analysis, infrared thermography, sound monitoring and ultrasound monitoring are some of the fault diagnostic techniques used in the industry to detect faults [2]. Each technique has its own advantage and is used for different applications. Among these, vibration monitoring technique continues to be used as a successful technique in fault diagnosis [3-5]. The vibration signals from REBs have many features that represent physical parameters to describe its functioning. The extraction of these features from the bearing vibrations, in order to arrive at a possible diagnosis of the working condition of REBs, is the main focus of a majority of recent research. The two main challenges in this approach are non-stationarity and the accumulation of noise in the obtained signals. The vibration that arises in bearings due to the misalignment of couplings and other machine components, or those generated from the working environment, is the main sources of noise [6]. Deconvolution of periodic impulse components $[7,8]$ and calculating spectral kurtosis $[9,10]$ are adopted to extract fault characteristics and diagnose early faults. As such noise is a major consideration when it comes to rotating machine condition monitoring and fault diagnosis.

The existing literature indicates various techniques used to extract features from vibration signals of REBs. One of the powerful methods for feature extraction is the wavelet transform. A review on the application of wavelet transform in machine fault diagnosis was presented by Yan et al. [11]. The identification of appropriate basis function by the wavelet transform for every defect-related signal

P. S. Ambika, ambikaps89@gmail.com | ${ }^{1}$ National Institute of Technology, Calicut, Calicut 673601 , India.

SN Applied Sciences (2019) 1:1020 | https://doi.org/10.1007/s42452-019-1005-3 
is a tedious task [12]. Empirical mode decomposition method (EMD) proposed by Huang et al. [13] is another recent technique for signal decomposition employing sifting architecture that decomposes a non-stationary signal into a predetermined number of modes or frequency components. Lei et al. [14] have summarized various works on bearing fault diagnosis employing the EMD method. However, as an empirical method, EMD, lacks a strong mathematical foundation and produces undesirable endeffects. Ensemble empirical mode decomposition (EEMD) method [15] helps in the reduction of mode mixing problem of the EMD algorithm. Empirical wavelet transform (EWT) [16] was later proposed, which partially addressed the drawbacks of EMD. It uses robust preprocessing for peak detection and appropriate wavelet filter banks for extracting the different modes of the original signal in the frequency domain, but with fixed bandwidth. Variational mode decomposition (VMD) [17] is a recent, fully adaptive algorithm, where mode selection can be performed by the user. It decomposes the signal into multiple modes with varying frequency content using the calculus of variation. VMD was used for the first time to detect multiple signatures caused by rotor-to-stator rubbing in 2015, by Wang et al. [18]. The filter bank property of VMD was also studied and tested [19]. VMD was shown to include more noise robustness and tone separation than EMD. It was also shown that statistical feature vectors from VMD are better than those obtained using EWT in SVM classification [20]. VMD was used in bearing fault analysis in [21-23] and the spreading in the frequency of the signal and intensity of vibration was demonstrated as a clear indication of a fault in bearings. VMD was also used in real-time power signal decomposition [24], brain magnetic resonance [25], ECG feature extraction and classification [26] and more recently in crude oil price analysis and forecasting [27]. VMD has also found applications in speech processing for the detection of voiced and non-voiced segments [28], instantaneous fundamental frequency [29] and estimation of glottal closure and opening instants in EGG [30]. However, the decomposition parameters in VMD are to be further manipulated for the study of REB fault diagnosis due to their non-stationary nature.

A hybrid model for denoising and bearing feature extraction using VMD was proposed recently by Zhang et al. [22]. Further study on compound fault diagnosis and characteristic separation of bearings and gearbox signals were carried out by optimizing the VMD algorithm [23]. In the present work, an attempt has been made to study the influence of initialization and input parameters (bandwidth selection parameter, $\alpha$ ) for the application pertaining to mechanical vibration signal processing. In such a scenario, condition monitoring of bearing vibration signals can be said to consist of oscillatory components, impulsive signals and underlying trends caused due to system dynamics. Hence certain parameters of the VMD algorithm need to be manipulated to suit this application $[18,22,23]$. Moreover, the signal decomposition in VMD can be deduced as wavelet packet like filter bank structure once the center frequencies of all the modes are initialized as zero. To further extract the relevant features of the mechanical vibration signals, distribution of energy in the available frequency components needs to be extracted.

The present work proposes a mode determination method by error values approach between BLIMFs and input signal. The error values calculated are MAE, RMSE and MAPE. The number of possible modes for the signal is estimated initially using EMD decomposition. The number of modes $(k)$ is tuned to $\alpha \pm 3$ for VMD. The mode number which attains the smallest error value average is selected as the mode number for an input signal. The present method is accurate, easy to implement, less complex and can provide accurate predictions to the mode determination of VMD. The main contributions of the present work are as follows:

- Initialization and input parameters of VMD algorithm are manipulated to be used in mechanical vibration signal processing.

- MAE, RMSE, MAPE are used as error indicators for mode determination.

- Run-to-failure vibration signature is used to characterize the behavior of bearing life.

- The algorithm is verified with existing works on bearing fault diagnosis and was found to perform better in terms of recognition rate and time taken.

\section{Materials and methods}

\subsection{Bearing test rig}

The procedure for fault diagnosis in this research work makes use of the IMS bearing prognosis data set. Here, bearing run-to-failure vibration signals are recorded from four Rexnord ZA-2115 double row bearings over durations of 30-35 days from a bearing prognostics test rig (Fig. 1). Each data set is a test-to-failure experiment on the bearings with individual files of 20,480 data points recorded at a sampling rate of $20 \mathrm{kHz}$.

Vibrations from bearing database are first decomposed into optimal modes using VMD. Then energy entropy of each IMF is computed as features from bearing vibrations. The extracted features are provided as input to an SVM multi-class classifier utilizing multiple kernel functions to determine their distinguishing capability in classifying faulty and non-faulty bearing signatures. Cross-validation 
Fig. 1 A schematic diagram of the bearing prognostics test rig

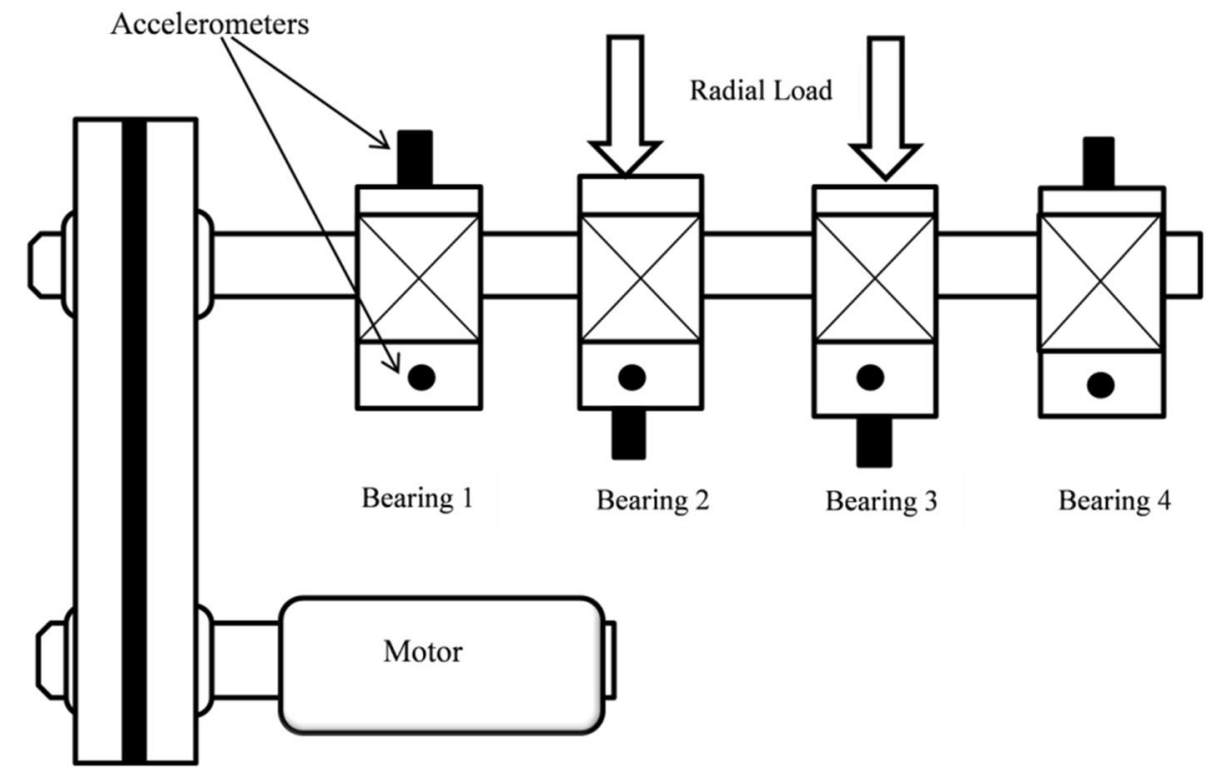

is also performed in SVM to select the best kernel parameters [31-34].

\subsection{Non-stationary signal decomposition with VMD}

The purpose of VMD is to decompose a multi-component signal $f(t)$ into a combination of a discrete set of quasiorthogonal IMFs [17]. Each mode of the signal is assumed to have a compact frequency support around a central frequency. VMD calculates these central frequencies and the IMFs centered on those frequencies concurrently using an optimization methodology called alternate direction method of multipliers (ADMM). The original formulation of the optimization problem is continuous in the time domain. Since part of the unknowns consists of functions, calculus of variation is applied to derive the optimal functions.

Each mode of the signal $u_{k}(t)$ is localized on a central frequency $\omega_{k}(t)$ which is calculated concurrently by VMD using the following constrained optimization technique [17]:

$\left.\begin{array}{l}\min _{u_{k}, \omega_{k}} \sum_{k}\left\|\partial_{t}\left[\left(\left(\delta(t)+\frac{j}{\pi t}\right) * u_{k}(t)\right) e^{-j \omega_{k} t}\right]\right\|_{2}^{2} \\ \text { such that } \sum_{k} u_{k}=f(t) .\end{array}\right\}$

where $f(t)$ is the signal, $u$ is its mode, $\omega$ is the frequency, $\delta$ is the dirac distribution, $t$ is the time script, $\partial_{t}$ is the time derivative, $k \in\{1,2, \ldots, K\}$ is the number of IMFs and * denotes convolution. The augmented Lagrangian multiplier method converts this into an unconstrained optimization problem as follows:

$$
\begin{aligned}
L\left(u_{k}, \omega_{k}, \lambda\right)= & \alpha \sum_{k}\left\|\partial_{t}\left[\left(\left(\delta(t)+\frac{j}{\pi t}\right) * u_{k}(t)\right) e^{-j \omega_{k} t}\right]\right\|_{2}^{2} \\
& +\left\|f-\sum_{k} u_{k}\right\|_{2}^{2}+\left\langle\lambda, f-\sum_{k} u_{k}\right\rangle
\end{aligned}
$$

where $\alpha$ is the balancing parameter of the data fidelity constraint and $\lambda$ is the Lagrangian multiplier.

The solution is obtained by solving for one variable at a time assuming all others to be known. Thus the IMFs $\widehat{u}_{k}(t)$ and the corresponding updated center frequency $\omega_{k}$ in the frequency domain can be obtained as:

$\hat{u}_{k}^{n+1}(\omega)=\frac{\hat{f}(\omega)-\sum_{i<k} \hat{u}_{i}^{n+1}(\omega)-\sum_{i>k} \hat{u}_{i}^{n}(\omega)+\frac{\hat{\lambda}^{n}(\omega)}{2}}{1+2 \alpha\left(\omega-\omega_{k}^{n}\right)^{2}}$.

$\omega_{k}^{n+1}=\frac{\int_{0}^{\infty} \omega\left|\hat{u}_{k}(\omega)\right|^{2} \mathrm{~d} \omega}{\int_{0}^{\infty}\left|\hat{u}_{k}(\omega)\right|^{2} \mathrm{~d} \omega}$

The uniqueness of the VMD algorithm lies in the assumption that each IMF of a 1-D signal has limited bandwidth. The calculation of bandwidth of the IMF $u_{k}(t)$ from the integration of the squared time derivative of the frequency translated signal can be written as follows:

$\Delta \omega_{k}=\int\left(\partial_{t}\left(u_{k}^{A}(t)\right) \times e^{-j \omega_{k} t}\right) \overline{\left(\partial_{t}\left(u_{k}^{A}(t)\right) \times e^{-j \omega_{k} t}\right)} \mathrm{d} t$, 
where

$u_{k}^{A}(t)=\left(u_{k}(t)+j u_{k}^{H}(t)\right)$.

$u_{k}^{A}(t)$ is the analytic signal representation of the IMF. $u_{k}^{H}(t)$ is the Hilbert transform of the IMF. The Hilbert transform $u_{k}^{H}(t)$ is calculated as the convolution of $u_{k}(t)$ and $1 / \pi t$, given by:

$u_{k}^{H}(t)=\frac{1}{\pi} \int_{-\infty}^{\infty} \frac{u_{k}(\tau)}{t-\tau} \mathrm{d} \tau$.

The integral in the above equation can also be expressed as a norm. Therefore, the bandwidth is obtained from the $L^{2}$-norm of the gradient of the demodulated signal as:

$$
\left.\begin{array}{rl}
\Delta \omega_{k} & =\left\|\partial_{t}\left[\left(\left(\delta(t)+\frac{j}{\pi t}\right) * u_{k}(t)\right) \times e^{-j \omega_{k} t}\right]\right\|_{2}^{2} \\
& =\partial_{t}\left\|\left(u_{k}(t)+j u_{k}^{H}(t)\right) \times e^{-j \omega_{k} t}\right\|_{2}^{2} \\
& =\partial_{t}\left\|u_{k}^{A}(t) \times e^{-j \omega_{k} t}\right\|_{2}^{2}
\end{array}\right\}
$$

Here, the term inside the gradient is the analytic signal representation of the mode $u_{k}(t)$ shifted to baseband frequencies by mixing with an exponential $e^{-j \omega t}$ tuned to the respective central frequencies $\omega_{k}$. The overview of the optimization algorithm of VMD is shown in Fig. 2. Manipulating parameters to suit a particular application is mandatory in data-driven approaches [22, 23]. Hence, alpha values are varied around a wide range $(100-1000000)$ and $k$ value between $(4,10)$ are used in the present work. Initialization of center frequencies is carried out in two ways: uniform distribution and zero. However, VMD shows good tone detection and noise robustness when compared to EMD [19]. The present approach attempts to investigate the influence of the main parameters of VMD in bearing fault diagnosis using a classification methodology called support vector machines (SVM). SVMs were initially designed for binary classifications which can be extended to multi-class classification using two basic approaches: combining several binary classifiers and the other by utilizing all data at once for optimization [35]. The first approach can be implemented using any of the following strategies: one-versus-all (using winner-takes-all strategy, WTA-SVM), one-versus-one (using max-wins voting, MWV-SVM), direct acyclic graph SVMs (DAGSVM) [36]. The all data at once approach is implemented in [37-40].

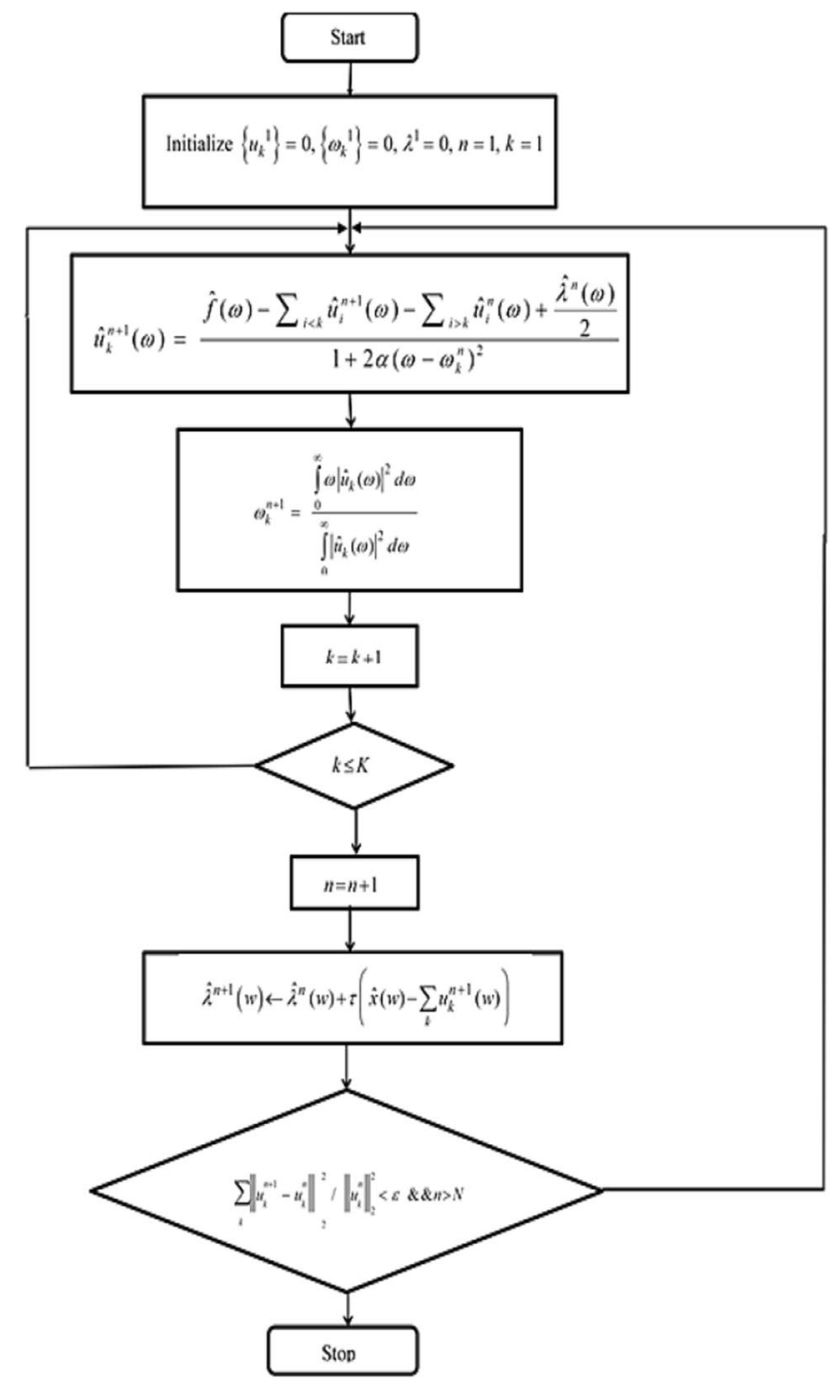

Fig. 2 An overview of the VMD optimization algorithm

\subsection{Extraction of energy entropy features}

The entropy feature extraction is based on the decomposition of the bearing signals using VMD. The band-limited IMFs contain sufficient frequency and amplitude-related information on bearing signals. Extraction of energy distribution-related features helps in determining fault information more precisely and thus distinguishing them from signals obtained during the healthy stage. The modes thus obtained are the BLIMFs from which the energy entropy features are extracted. This forms the feature matrix which is input to the multi-class SVM classifier for training and testing the classifier to obtain the accuracy percentages with which each class of feature matrices are distinguished. The energy entropy is defined as:

$E_{E N}=-h_{k} \log h_{k}$ 
where $h_{k}=E_{k} / E$, the ratio of energy of each IMF to the total energy $\left(E=\Sigma E_{k}\right)$. The VMD-based entropy feature extraction includes VMD-based decomposition, energy entropy estimations and classification.

\subsection{Principle analysis of SVM method}

The basic theory of SVM method has been elaborated. The main idea here is to map the signal to a higher dimensional space and find an optimal hyperplane in the space that maximizes the distance between the classes. The hyperplane $h$ is given by [35]:

$y=h(x, w)=\langle w, x\rangle+b$

Normally, SVM classifiers are used for binary classifications. In the present work, the SVM classifier uses the one-against-all approach for multi-class classification. It constructs $m$ binary SVM models where $m$ is the number of classes. The $m$ th SVM is trained with all of the samples in $m$ th class with positive labels and all other classes with negative labels $[41,42]$. If $\left(x_{i}, y_{i}\right)$ are the training samples of data label pairs where $x_{i} \in \mathbb{R}^{n}$ and $i=(1,2, \ldots, n)$ where $n$ is the number of data points and $y=\{-1,1\}$ are the labels. The formulation for binary SVM classification goes:

$$
\left.\begin{array}{c}
\min _{w, b, \zeta} \frac{1}{2} w, w+C \sum_{i=1}^{n} \zeta_{i} \\
\text { subject to } \quad y_{i} \cdot\left(w \cdot x_{i}+b\right) \geq 1-\zeta_{i} \\
\zeta_{i} \geq 0 \quad \text { and } \quad i=1,2, \ldots, n .
\end{array}\right\}
$$

where $w_{i}, b \in \mathbb{R}^{n}$ are the weighting factors, $\zeta$ is the slack variable giving a soft classification boundary and $C=1 / \lambda n$ is the penalty parameter which gives a trade-off between the range of fault patterns and the number of fault samples rejected. $w$ is the hyperplane in $\mathbb{R}^{n}$ that separates the classes. Hence, for linear separation, it is necessary to map the training samples $x_{i}$ into a higher dimensional space:

$y=h(x, w)=w, \phi(x)+b$

where $\phi($.$) represents a mapping from input space to a$ feature space $F$. A generalized SVM function, therefore, uses a kernel function $K$, which helps in transforming the training samples $\left\{\left(x_{1}, y_{1}\right),\left(x_{2}, y_{2}\right), \ldots,\left(x_{n}, y_{n}\right)\right\}$ to a higher dimensional space to allow linear separation of input space parameters using the dot product:

$K\left(x_{i}, x_{j}\right)=\phi\left(x_{i}\right), \phi\left(x_{j}\right)$

For a more detailed theory of kernel functions and the evolution of SVM, the reader is directed to the text book on support vector machines by Soman et al. [27]. Based on this, the different kernels employed in the present study are:

- $\operatorname{Linear} K\left(x_{i}, x_{j}\right)=x_{i} T, x_{j}$

- Polynomial $K\left(x_{i}, x_{j}\right)=\left(\gamma x_{i}^{T}, x_{j}+r\right)^{d} \gamma>0$

- Radial basis function (RBF)

$K\left(x_{i}, x_{j}\right)=\exp \left(-\gamma\left\|x_{i}-x_{j}\right\|^{2}\right), \quad \gamma>0$

where $K\left(x_{i}, x_{j}\right)=\phi\left(x_{i}^{T}\right), \phi\left(x_{j}\right)$ and $\gamma, d$ are kernel parameters. The overall fault diagnosis approach is shown in Fig. 3.

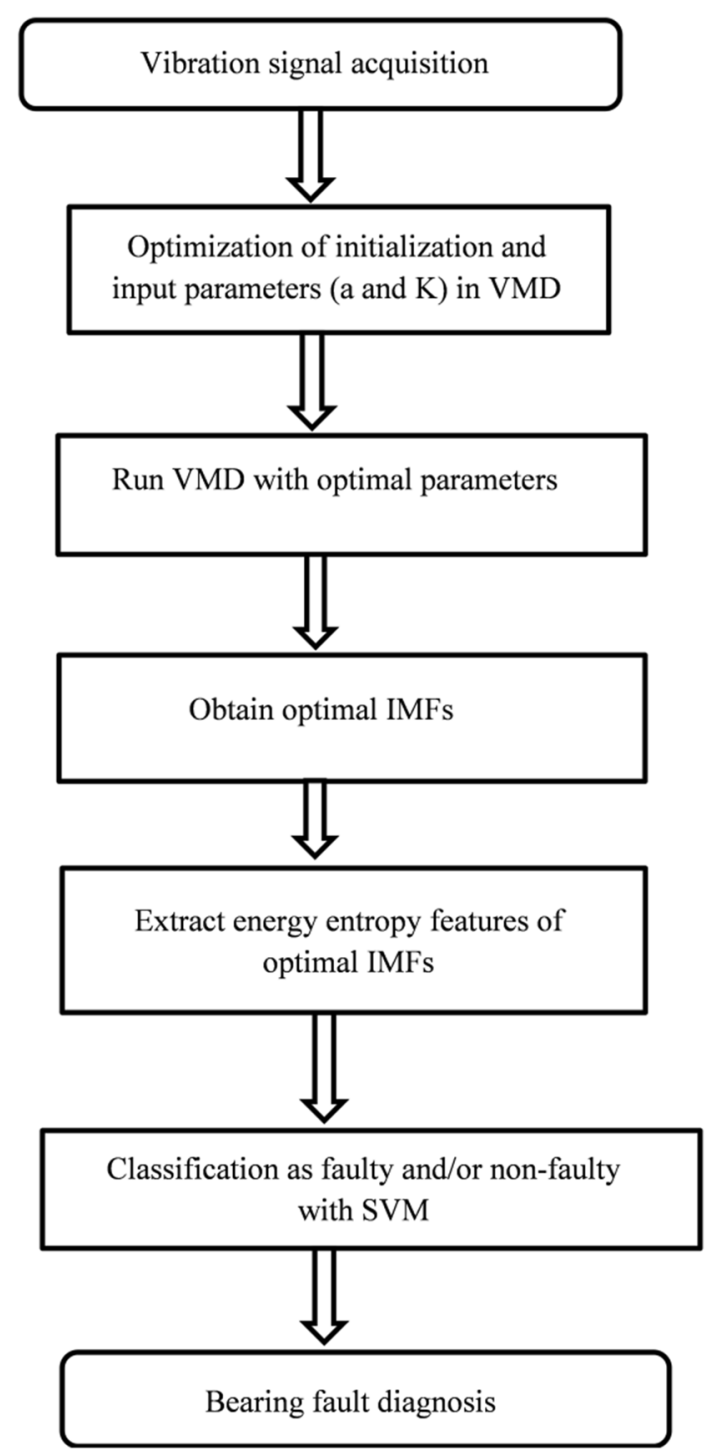

Fig. 3 The overall procedure for the bearing fault diagnosis approach 


\section{Experimental results}

The experiments are carried out in three phases.

1. Empirically fixing the tuning parameters of VMD.

2. Extracting energy entropy features from vibration signals.

3. Classifying the features with SVM cross-validation.

Each of the above steps is described below in detail.

\subsection{Selecting the VMD input parameters}

The input parameters for VMD algorithm such as bandwidth selection balancing parameter, $\alpha$ (alpha) and the number of modes, $k$ are fixed based on error obtained from input vibration signal (actual signal) and sum of modes obtained from VMD for input parameters varying along the range $a=(100,500,1500,3000,5000,10000$, $50000,100000,1000000)$ and $k=(4,5,6,7,8,9,10)$ and initialization parameter ranging between $(0,1)$. The error values are obtained from performance measures such as:
- Mean Absolute Error (MAE) Mean of the absolute value of the difference between actual and predicted signals.

- Root Mean Square Error (RMSE) Root of the mean value of the squared difference between actual and predicted signal values.

- Mean Absolute Percentage Error (MAPE) Mean of the absolute value of the percentage of difference between actual and predicted signals.

From the above measures, mean absolute error (MAE) is regarded as the mode determination parameter for selecting the VMD input parameters. The alpha $(\alpha)$ and number of modes $(k)$ are determined from the combination where MAE is the least. The results are depicted in Tables 1 and 2. When the initialization parameter is set to zero, the MAE value reduces for increasing alpha (Table 1). The lowest error value is obtained for alpha, $a=1000000$. The error values are lowest for all the $k$ values. We therefore select $k=5$ for our experiments. When the initialization parameter is unity, a similar trend is observed for the error values. For our experiments, therefore, we choose $a=1,000,000$ and $k=5$. The time taken for decomposing a signal into $k=5$ modes

Table 1 Mean absolute error rate (MAE) for faulty signal at initialization parameter init $=0$, and number of modes $k=(4,10)$ and balancing parameter $a=(100,1000000)$

\begin{tabular}{|c|c|c|c|c|c|c|c|c|c|c|c|c|}
\hline & & \multicolumn{11}{|c|}{ Alpha (a) } \\
\hline & & 100 & 500 & 1500 & 3000 & 5000 & 7500 & 10,000 & 20,000 & 50,000 & 100,000 & $1,000,000$ \\
\hline \multirow{7}{*}{ 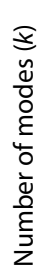 } & $k=4$ & 0.1406 & 0.1370 & 0.1269 & 0.1217 & 0.1145 & 0.1144 & 0.1114 & 0.1100 & 0.1087 & 0.1026 & 0.1002 \\
\hline & $k=5$ & 0.1410 & 0.1381 & 0.1335 & 0.1221 & 0.1151 & 0.1152 & 0.1149 & 0.1093 & 0.1089 & 0.1027 & 0.1002 \\
\hline & $k=6$ & 0.1414 & 0.1391 & 0.1354 & 0.1305 & 0.1210 & 0.1159 & 0.1153 & 0.1144 & 0.1105 & 0.1078 & 0.1003 \\
\hline & $k=7$ & 0.1421 & 0.1398 & 0.1368 & 0.1327 & 0.1283 & 0.1209 & 0.1160 & 0.1164 & 0.1106 & 0.1078 & 0.1003 \\
\hline & $k=8$ & 0.1423 & 0.1401 & 0.1378 & 0.1349 & 0.1307 & 0.1273 & 0.1205 & 0.1206 & 0.1117 & 0.1079 & 0.1004 \\
\hline & $k=9$ & 0.1424 & 0.1406 & 0.1382 & 0.1355 & 0.1332 & 0.1298 & 0.1229 & 0.1159 & 0.1118 & 0.1104 & 0.1004 \\
\hline & $k=10$ & 0.1424 & 0.1407 & 0.1388 & 0.1363 & 0.1339 & 0.1308 & 0.1240 & 0.1159 & 0.1118 & 0.1104 & 0.1004 \\
\hline
\end{tabular}

Table 2 Mean absolute error rate (MAE) for faulty signal at initialization parameter init $=1$, and number of modes $k=(4,10)$ and balancing parameter $a=(100,1000000)$

\begin{tabular}{|c|c|c|c|c|c|c|c|c|c|c|c|}
\hline & & \multicolumn{10}{|c|}{ Alpha (a) } \\
\hline & & 100 & 500 & 1500 & 3000 & 5000 & 7500 & 10,000 & 50,000 & 100,000 & $1,000,000$ \\
\hline \multirow{7}{*}{ 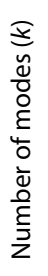 } & $k=4$ & 0.1406 & 0.1356 & 0.1301 & 0.1261 & 0.1226 & 0.1204 & 0.1191 & 0.1040 & 0.1024 & 0.1003 \\
\hline & $k=5$ & 0.1410 & 0.1382 & 0.1336 & 0.1238 & 0.1213 & 0.1195 & 0.1184 & 0.1078 & 0.1069 & 0.1030 \\
\hline & $k=6$ & 0.1412 & 0.1387 & 0.1341 & 0.1301 & 0.1270 & 0.1246 & 0.1231 & 0.1091 & 0.1059 & 0.1028 \\
\hline & $k=7$ & 0.1414 & 0.1390 & 0.1359 & 0.1305 & 0.1279 & 0.1254 & 0.1241 & 0.1119 & 0.1048 & 0.1028 \\
\hline & $k=8$ & 0.1415 & 0.1398 & 0.1340 & 0.1305 & 0.1272 & 0.1249 & 0.1235 & 0.1112 & 0.1095 & 0.1027 \\
\hline & $k=9$ & 0.1423 & 0.1404 & 0.1370 & 0.1337 & 0.1309 & 0.1288 & 0.1274 & 0.1184 & 0.1143 & 0.1181 \\
\hline & $k=10$ & 0.1423 & 0.1407 & 0.1378 & 0.1347 & 0.1322 & 0.1302 & 0.1289 & 0.1171 & 0.1148 & 0.1074 \\
\hline
\end{tabular}


with $a=1000000$ is almost the same as that for decomposing with $a=100$. The time taken for decomposing a signal with $k=5, a=1000000$ is $160.71 \mathrm{~s}$, whereas the time it takes to decompose a signal with $k=5, a=100$ is $167.87 \mathrm{~s}$.

\subsection{Fixing the initialization and input parameters for vibration classification}

In this application, vibration signals from three bearings ending with a faulty roller, faulty inner race and faulty outer race with 2155, 2155 and 984 records, respectively, are used. The records from a bearing constitute the entire run-to-failure vibration data of that bearing with 20,480 samples per second. Each bearing has three zones of operating conditions namely healthy, degrading and faulty. Taking into consideration the overlap between the zones, 3100 signals were carefully extracted from the three zones of the bearings. Thus, the data matrix now has seven classes of bearing vibration signals: healthy $(H)$, degrading inner race (DIR), degrading outer race (DOR), degrading roller (DR), faulty inner race (FIR), faulty outer race (FOR) and faulty roller (FR). This matrix of vibration signatures is provided as input to VMD to obtain IMFs, whose center frequencies (omega, $\omega$ ) are initialized in two ways: All omegas start at zero. All omegas start uniformly distributed.

For each initialization, the input parameter alpha $(a)$ is varied between 100 and 1000000 keeping $D C=0$ and tolerance $\tau=0$ for convergence criterion as $1 e^{-7}$. The decomposition results achieved using VMD for
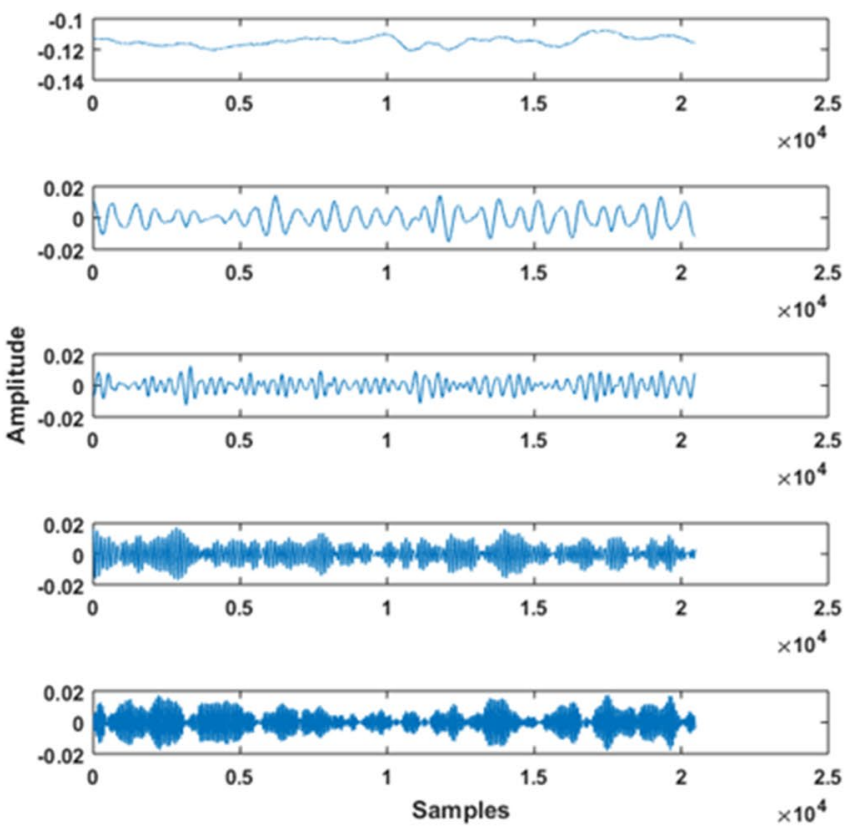

$a=1000000$ and initialization parameter set at zero for a healthy and faulty signal are shown in Figs. 4 and 5 . Energy entropy features are extracted from the IMFs as elaborated in Sect. 2.3. The feature matrix for 12 modes of a signal from each class showing the distribution of energy entropy values is given in Table 1. This feature matrix is provided as input to the SVM classifier which outputs the label of the unknown classes for three different kernel functions. From the output obtained by SVM, the generalized classification accuracy can also be calculated from the ratio of correctly classified test signatures to the total number of test signatures.

\subsection{Bearing run-to-failure database}

The IMS bearing database [43] consists of three sets of four- and eight-channel 1-second vibration signal snapshots recorded from four Rexnord ZA-2115 double row bearings over durations of 30-35 days. Each data set is a test-to-failure experiment on the bearings with individual files of 20,480 data points recorded at a sampling rate of $20 \mathrm{kHz}$. Each file is recorded at specific intervals, and the file name indicates the time when the data was collected.

\subsection{Classification of features using SVM cross-validation}

The classification of bearing signatures into faulty and non-faulty with SVM requires a multi-class classification
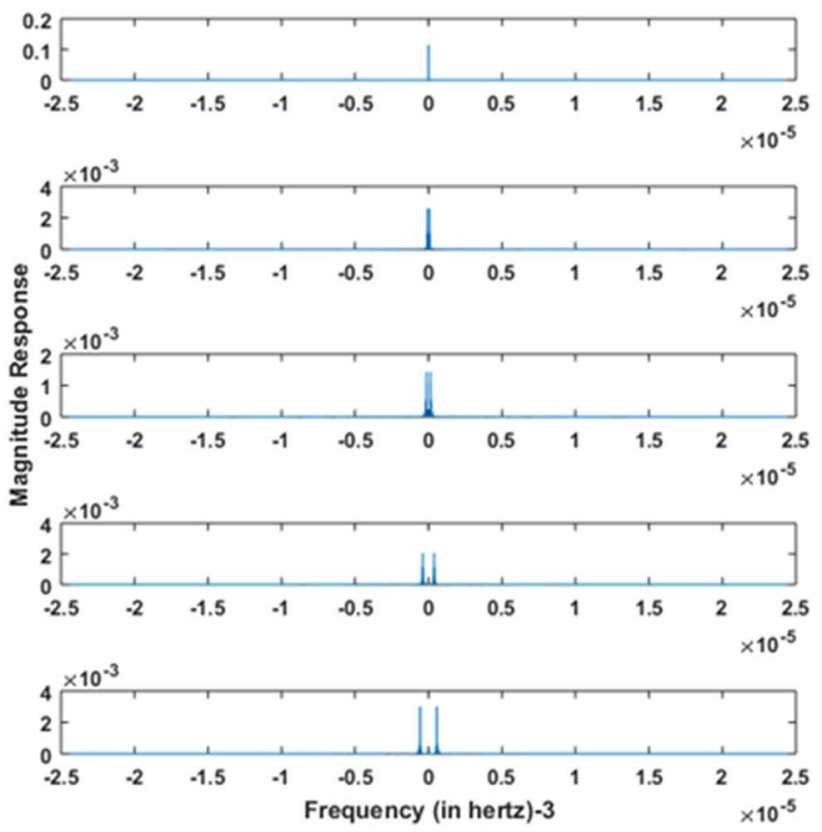

Fig. 4 The modes and corresponding frequency spectra of healthy signal decomposed into $k=5$ number of modes for balancing parameter $a=1,000,000$ 

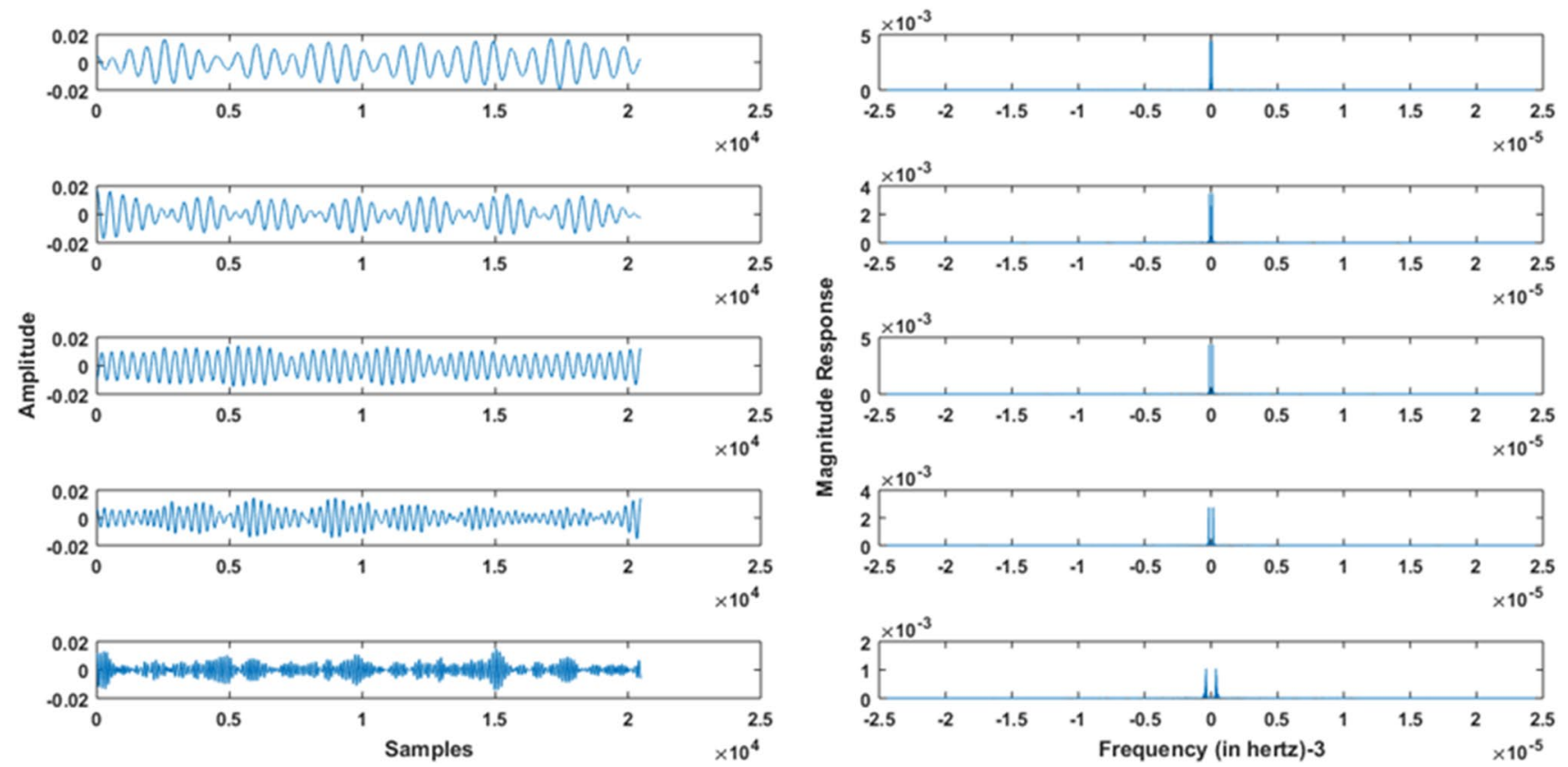

Fig. 5 The modes and corresponding frequency spectra of faulty signal decomposed into $k=5$ number of modes for balancing parameter $a=1,000,000$

Table 3 A comparison of time taken (in seconds) by SVM using RBF kernel for initialization parameter zero where all omegas start at zero

\begin{tabular}{llllll}
\hline$(C, \gamma)$ & One-against-one & & & One-against-all & \\
\cline { 2 - 3 } & Accuracy (\%) & $\begin{array}{l}\text { Training and testing } \\
\text { time taken }(\mathrm{s})\end{array}$ & & Accuracy (\%) & $\begin{array}{l}\text { Training and test- } \\
\text { ing time taken (s) }\end{array}$ \\
\hline$\left(2^{14}, 2\right)$ & 0.96 & 17.63 & 0.88 & 2.71 \\
$\left(2^{4}, 4\right)$ & 0.96 & 17.28 & 0.92 & 1.97 \\
$\left(2^{11}, \mathbf{2}\right)$ & 1.0 & 17.29 & & 0.90 & 1.93 \\
$\left(2^{8}, 4\right)$ & 0.90 & 19.29 & 0.90 & 1.83 \\
\hline
\end{tabular}

strategy. This is carried out using one-against-one and one-against-all approach. The former decomposes the $m$-class problem into a series of two-class problems and constructs $m(m-1) / 2$ classifiers, where $m$ is the number of classes. SVM forms $m$ classifiers in the later. Here, the $m$ th class is trained with positive labels and all other classes with negative labels. The training samples $\left\{\left(x_{1}, y_{1}\right),\left(x_{2}, y_{2}\right), \ldots,\left(x_{n}, y_{n}\right)\right\}$ are mapped to a higher dimensional space by the kernel function and therefore can handle the case when the relation between the class label and training sample is nonlinear. Three kernel functions are utilized to obtain generalized accuracy as mentioned in Sect. 3.2. In addition, cross-validation is carried out using LIBSVM [44] to select the best possible values of $(C, \gamma)$. Various combinations of $(C, \gamma)$ are tried, and the one with the best validation performance is selected. Exponentially growing sequences of $(C, \gamma)$ are utilized; for example: $C=\left(2^{-5}, 2^{-13}, \ldots, 2^{3}\right)$ and $\gamma=\left(2^{-15}, 2^{-13}, \ldots, 2^{3}\right)$. Thus, $18 \times 17$ combinations are tried. $70 \%$ instances from the data matrix are grouped as train data and the remaining as test data for fivefold cross-validation. Then the train data are grouped into five equal groups, where each group is tested on SVM trained on all other groups. In this manner, the cross-validation is able to find the best possible parameters $(C, \gamma)$. These are utilized to train the entire training set once again. After finding the optimal parameters, the classifier predicts the generalized accuracy for the unknown test data set. If many $(C, \gamma)$ have the best validation rate, apply them all to the test data and take the highest [44].

Tables 3 and 4 show the classification results from SVM classifier using the RBF kernel. The initialization parameters are varied for alpha values ranging from 100 to 1000000 . The relevant results are tabularized. The highest accuracy 
values obtained are shown in bold. When compared to the one-against-one approach, the one-against-all approach provides relatively lesser recognition of faulty and nonfaulty classes. The corresponding $(C, \gamma)$ values are also given. The average time taken by the RBF kernel during training and testing phases together is reported in Tables 4 and 5 . The confusion matrix obtained for initialization parameter zero, when alpha is 2500 and $(C, \gamma)$ values are $\left(2^{11}, 2^{2}\right)$, is shown in Table 5.

\section{Comparison with existing works}

The VMD-based feature extraction has been carried out on bearing signals obtained from both SpectraQuest MFS database and University of Cincinnati IMS database to obtain fault-related features from real-time bearing vibration signals decomposed into IMFs. The energy entropy characteristics of the signals were used as features. As the VMD algorithm decomposes each signal into IMFs of different frequency components, energy distribution can be studied in further detail. Fault identification features were
Table 4 A comparison of generalized accuracy (\%) using RBF kernel for uniform initialization parameter where all omegas are uniformly distributed

\begin{tabular}{|c|c|c|c|c|}
\hline \multirow[t]{2}{*}{$(C, \gamma)$} & \multicolumn{2}{|c|}{ One-against-one } & \multicolumn{2}{|c|}{ One-against-all } \\
\hline & Accuracy (\%) & $\begin{array}{l}\text { Training and testing } \\
\text { time taken (s) }\end{array}$ & Accuracy (\%) & $\begin{array}{l}\text { Training and test- } \\
\text { ing time taken (s) }\end{array}$ \\
\hline$\left(2^{9}, 4\right)$ & 1.0 & 18.19 & 0.96 & 2.55 \\
\hline$\left(2^{5}, 4\right)$ & 0.94 & 17.83 & 0.96 & 2.80 \\
\hline$\left(2^{9}, 2\right)$ & 0.96 & 17.84 & 0.94 & 2.69 \\
\hline$\left(2^{8}, 2\right)$ & 0.92 & 17.02 & 0.96 & 1.99 \\
\hline
\end{tabular}

Table 5 Confusion matrix for SVM using VMD-based entropy features

\begin{tabular}{lcllllll}
\hline $\mathrm{H}$ & DR & DIR & DOR & FR & FIR & FOR & \\
\hline 4 & 0 & 0 & 0 & 0 & 0 & 0 & H \\
0 & 10 & 0 & 0 & 0 & 0 & 0 & DR \\
0 & 0 & 3 & 0 & 0 & 0 & 0 & DIR \\
0 & 0 & 0 & 8 & 0 & 0 & 0 & DOR \\
0 & 0 & 0 & 0 & 6 & 0 & 0 & FR \\
0 & 0 & 0 & 0 & 0 & 7 & 0 & FIR \\
0 & 0 & 0 & 0 & 0 & 0 & 12 & FOR \\
\hline
\end{tabular}

$H$ healthy, $D R$ degrading roller, DIR degrading inner race, DOR degrading outer race, FR faulty roller, FIR faulty inner race, FOR faulty outer race

Table 6 Comparison of the proposed method with existing literature

\begin{tabular}{|c|c|c|c|c|c|}
\hline & \multicolumn{5}{|l|}{ References } \\
\hline & Zhang et. al [45] & Kankar et al. [46] & Vakharia et al. [47] & Liu et al. [48] & Present work \\
\hline $\begin{array}{l}\text { Method used for } \\
\text { vibration signal } \\
\text { analysis }\end{array}$ & $\begin{array}{l}\text { EEMD and permuta- } \\
\text { tion entropy of each } \\
\text { IMF }\end{array}$ & $\begin{array}{l}\text { Continuous wavelet } \\
\text { transform (Meyer } \\
\text { wavelet) }\end{array}$ & $\begin{array}{l}\text { Multi-scale permuta- } \\
\text { tion entropy and } \\
\text { wavelet }\end{array}$ & $\begin{array}{l}\text { EMD and } \\
\text { statistical } \\
\text { characteristics } \\
\text { of IMFs }\end{array}$ & $\begin{array}{l}\text { VMD and energy } \\
\text { entropy characteris- } \\
\text { tics of IMFs }\end{array}$ \\
\hline Classifier used & $\begin{array}{l}\text { SVM with parameter } \\
\text { optimization by Inter } \\
\text { Cluster Distance }\end{array}$ & $\begin{array}{l}\text { SVM with } 10 \text {-fold } \\
\text { cross-validation }\end{array}$ & SVM & $\begin{array}{l}\text { Wavelet sup- } \\
\text { port vector } \\
\text { machine } \\
\text { (WSVM) }\end{array}$ & $\begin{array}{l}\text { SVM with fivefold cross- } \\
\text { validation }\end{array}$ \\
\hline No. of classes & 4; H, IRF, ORF, RF & 5; H, IRF, ORF, RF, CF & 4; H, IRF, ORF, RF & $4 ; \mathrm{H}, \mathrm{IRF}, \mathrm{ORF}, \mathrm{RF}$ & $\begin{array}{l}\text { 7; H, DR, DIR, DOR, FR, } \\
\text { FIR, FOR }\end{array}$ \\
\hline Classification rate (\%) & $97.91-100$ & 98.67 & 97.5 & 97.5 & $98.67-100$ \\
\hline Remarks & & & & & $\begin{array}{l}\text { Run-to-failure data set } \\
\text { of bearing vibration } \\
\text { signal is utilized and } \\
\text { detect rate is } 100 \%\end{array}$ \\
\hline
\end{tabular}


obtained on the extraction of energy distribution features from the healthy and faulty signals. The classification accuracy obtained from the proposed method is compared with that obtained with existing works in Table 6.

\section{Conclusion}

In the present work, VMD-based feature extraction method is evaluated on seven classes of bearing signatures. The multi-class SVM algorithm is employed for classification. After testing, the best accuracy results are obtained at $100 \%$ for RBF kernel with optimal kernel parameters. This shows that adaptively varying the parameters pertaining to VMD algorithm provides better results in bearing signature fault diagnosis. From the analysis and experimental results, it is concluded that:

1. The frequency components (BLIMFs) decomposed by VMD can detect fault propagation in rolling element bearings.

2. Energy entropy feature extraction from the VMD successfully identified the working condition and fault patterns and provided a useful tool for intelligent fault diagnosis of rolling element bearings.

3. Cross-validating the RBF kernel parameters in SVM multi-class classification gives promising results in rolling element bearing condition monitoring.

Acknowledgements The authors would like to thank the Center for Intelligent Maintenance Systems (IMS), University of Cincinnati, USA for providing the bearing dataset. The authors would also like to express their hearfelt gratitude to Dr. K. P. Soman, Mr. Sachin Kumar and Ms. Neethu Mohan (Amrita University, Coimbatore), for their valuable comments and suggestions.

\section{Compliance with ethical standards}

Conflict of interest The authors declare that they have no conflict of interest.

\section{References}

1. Randall RB (2011) Vibration-based condition monitoring: industrial, aerospace and automotive applications. Wiley, New York

2. Edwards S, Lees AW, Friswell MI (1998) Fault diagnosis of rotating machinery. Shock Vib Dig 30(1):4-13

3. Taylor Jl (1995) Back to the basics of the rotating machinery vibration analysis. Sound Vib 29(2):12-16

4. Renwick JT, Babson PE (1985) Vibration analysis-a proven technique as a predictive maintenance tool. IEEE Trans Ind Appl 2:324-332. https://doi.org/10.1109/TIA.1985.349652
5. Fan X, Zuo MJ (2008) Machine fault feature extraction based on intrinsic mode functions. Meas Sci Technol 19(4):045105

6. Yu L, Junhong Z, Fengrong B, Jiewei L, Wenpeng M (2014) A fault diagnosis approach for diesel engine valve train based on improved ITD and SDAG-RVM. Meas Sci Technol 26(2):025003

7. Miao Y, Zhao M, Lin J, Xu X (2016) Sparse maximum harmonicsto-noise-ratio deconvolution for weak fault signature detection in bearings. Meas Sci Technol 27(10):105004

8. Miao Y, Zhao M, Lin J, Lei Y (2017) Application of an improved maximum correlated kurtosis deconvolution method for fault diagnosis of rolling element bearings. Mech Syst Signal Process 92:173-195. https://doi.org/10.1016/j.ymssp.2017.01.033

9. Antoni J (2006) The spectral kurtosis: a useful tool for characterising non-stationary signals. Mech Syst Signal Process 20(2):282307. https://doi.org/10.1016/j.ymssp.2004.09.001

10. Miao Y, Zhao M, Lin J (2017) Improvement of kurtosis-guidedgrams via Gini index for bearing fault feature identification. Meas Sci Technol 28(12):125001

11. Yan R, Gao RX, Chen X (2014) Wavelets for fault diagnosis of rotary machines: a review with applications. Sig Process 96:1-15. https://doi.org/10.1016/j.sigpro.2013.04.015

12. Soman KP (2010) Insight into wavelets: from theory to practice. PHI Learning Pvt. Ltd., Delhi

13. Huang NE, Shen Z, Long SR, Wu MC, Shih HH, Zheng Q, Yen NC, Tung CC, Liu HH (1998) The empirical mode decomposition and the Hilbert spectrum for nonlinear and non-stationary time series analysis. Proc R Soc Lond Ser A Math Phys Eng Sci 454(1971):903-995. https://doi.org/10.1098/rspa.1998.0193

14. Lei Y, Lin J, He Z, Zuo MJ (2013) A review on empirical mode decomposition in fault diagnosis of rotating machinery. Mech Syst Signal Process 35(1-2):108-126. https://doi.org/10.1016/j. ymssp.2012.09.015

15. Guo W, Peter WT (2010) Enhancing the ability of ensemble empirical mode decomposition in machine fault diagnosis. In: 2010 Prognostics and system health management conference. IEEE, pp 1-7. https://doi.org/10.1109/phm.2010.5413421

16. Gilles J (2013) Empirical wavelet transform. IEEE Trans Signal Process 61(16):3999-4010. https://doi.org/10.1109/ TSP.2013.2265222

17. Dragomiretskiy K, Zosso D (2013) Variational mode decomposition. IEEE Trans Signal Process 62(3):531-544. https://doi. org/10.1109/TSP.2013.2288675

18. Wang Y, Markert R, Xiang J, Zheng W (2015) Research on variational mode decomposition and its application in detecting rub-impact fault of the rotor system. Mech Syst Signal Process 60:243-251. https://doi.org/10.1016/j.ymssp.2015.02.020

19. Wang $Y$, Markert R (2016) Filter bank property of variational mode decomposition and its applications. Sig Process 120:509-521. https://doi.org/10.1016/j.sigpro.2015.09.041

20. Aneesh C, Kumar S, Hisham PM, Soman KP (2015) Performance comparison of variational mode decomposition over empirical wavelet transform for the classification of power quality disturbances using support vector machine. Procedia Comput Sci 46:372-380. https://doi.org/10.1016/j.procs.2015.02.033

21. Gupta KK, Raju KS (2014) Bearing fault analysis using variational mode decomposition. In: 2014 9th International conference on industrial and information systems (ICIIS). IEEE, pp 1-6. https://doi.org/10.1109/ICIINFS.2014.7036617

22. Zhang $S$, Wang $Y$, He S, Jiang Z (2016) Bearing fault diagnosis based on variational mode decomposition and total variation denoising. Meas Sci Technol 27(7):075101

23. Yan X, Jia M, Xiang L (2016) Compound fault diagnosis of rotating machinery based on OVMD and a 1.5-dimension envelope spectrum. Meas Sci Technol 27(7):075002

24. Soman KP, Poornachandran P, Athira S, Harikumar K (2015) Recursive variational mode decomposition algorithm for real 
time power signal decomposition. Procedia Technol 21:540546. https://doi.org/10.1016/j.protcy.2015.10.048

25. Lahmiri S (2016) Image characterization by fractal descriptors in variational mode decomposition domain: application to brain magnetic resonance. Physica A 456:235-243. https:// doi.org/10.1016/j.physa.2016.03.046

26. Mert A (2016) ECG feature extraction based on the bandwidth properties of variational mode decomposition. Physiol Meas 37(4):530

27. Jianwei E, Bao Y, Ye J (2017) Crude oil price analysis and forecasting based on variational mode decomposition and independent component analysis. Physica A 484:412-427. https:// doi.org/10.1016/j.physa.2017.04.160

28. Upadhyay A, Pachori RB (2015) Instantaneous voiced/nonvoiced detection in speech signals based on variational mode decomposition. J Frankl Inst 352(7):2679-2707. https://doi. org/10.1016/j.jfranklin.2015.04.001

29. Upadhyay A, Sharma M, Pachori RB (2017) Determination of instantaneous fundamental frequency of speech signals using variational mode decomposition. Comput Electr Eng 62:630647. https://doi.org/10.1016/j.compeleceng.2017.04.027

30. Lal GJ, Gopalakrishnan EA, Govind D (2018) Accurate estimation of glottal closure instants and glottal opening instants from electroglottographic signal using variational mode decomposition. Circuits Syst Signal Process 37(2):810-830. https://doi. org/10.1007/s00034-017-0582-x

31. Sajid M, lqbal Ratyal N, Ali N, Zafar B, Dar SH, Mahmood MT, Joo YB (2019) The impact of asymmetric left and asymmetric right face images on accurate age estimation. Math Probl Eng. https ://doi.org/10.1155/2019/8041413

32. Ratyal NI, Taj IA, Sajid M, Ali N, Mahmood A, Razzaq S (2019) Three-dimensional face recognition using variance-based registration and subject-specific descriptors. Int J Adv Rob Syst 16(3):1729881419851716. https://doi.org/10.1177/1729881419 851716

33. Zafar B, Ashraf R, Ali N, Iqbal M, Sajid M, Dar S, Ratyal N (2018) A novel discriminating and relative global spatial image representation with applications in CBIR. Appl Sci 8(11):2242. https ://doi.org/10.3390/app8112242

34. Ali N, Zafar B, Riaz F, Dar SH, Ratyal NI, Bajwa KB, lqbal MK, Sajid $M$ (2018) A hybrid geometric spatial image representation for scene classification. PLoS ONE 13(9):e0203339. https://doi. org/10.1371/journal.pone.0203339

35. Soman KP, Loganathan R, Ajay V (2009) Machine learning with SVM and other kernel methods. PHI Learning Pvt. Ltd., Delhi
36. Hsu CW, Lin CJ (2002) A comparison of methods for multiclass support vector machines. IEEE Trans Neural Netw 13(2):415-425

37. Duan KB, Keerthi SS (2005) Which is the best multiclass SVM method? An empirical study. In: International workshop on multiple classifier systems. Springer, Berlin, pp 278-285. https://doi. org/10.1007/11494683_28

38. Crammer K, Singer $Y$ (2002) On the learnability and design of output codes for multiclass problems. Mach Learn 47(2-3):201233. https://doi.org/10.1023/A:1013637720281

39. Vapnik V, Vapnik V (1998) Statistical learning theory. Wiley, New York, pp 156-160

40. Weston J, Watkins C (1999) Support vector machines for multiclass pattern recognition. In: Esann, vol 99, pp 219-224

41. Hsu CW, Chang CC, Lin CJ (2003) A practical guide to support vector classification. Tech. rep., Department of Computer Science, National Taiwan University

42. Dybała J, Zimroz R (2014) Rolling bearing diagnosing method based on empirical mode decomposition of machine vibration signal. Appl Acoust 77:195-203. https://doi.org/10.1016/j.apaco ust.2013.09.001

43. Qiu H, Lee J, Lin J, Yu G (2006) Wavelet filter-based weak signature detection method and its application on rolling element bearing prognostics. J Sound Vib 289(4-5):1066-1090. https:// doi.org/10.1016/j.jsv.2005.03.007

44. Chang CC (2011) LIBSVM: a library for support vector machines. ACM Trans Intell Syst Technol 2:27

45. Zhang $X$, Liang Y, Zhou J (2015) A novel bearing fault diagnosis model integrated permutation entropy, ensemble empirical mode decomposition and optimized SVM. Measurement 69:164-179. https://doi.org/10.1016/j.measuremen t.2015.03.017

46. Kankar PK, Sharma SC, Harsha SP (2011) Fault diagnosis of ball bearings using continuous wavelet transform. Appl Soft Comput 11(2):2300-2312. https://doi.org/10.1016/j.asoc.2010.08.011

47. Vakharia V, Gupta VK, Kankar PK (2015) Ball bearing fault diagnosis using supervised and unsupervised machine learning methods. Int J Acoust Vib 20(4):244-250

48. Liu Z, Cao H, Chen X, He Z, Shen Z (2013) Multi-fault classification based on wavelet SVM with PSO algorithm to analyze vibration signals from rolling element bearings. Neurocomputing 99:399-410. https://doi.org/10.1016/j.neucom.2012.07.019

Publisher's Note Springer Nature remains neutral with regard to jurisdictional claims in published maps and institutional affiliations. 